\title{
Zur Theorie der Sättigungsmagnetisierung in binären ferromagnetischen Legierungen
}

\author{
Von Hermann Statz \\ Aus dem Institut für theoretische und angewandte Physik der \\ Technischen Hochschule Stuttgart
}

(Z. Naturforschg. 7 a, 506-511 [1952]; eingegangen am 24. Mai 1952)

\begin{abstract}
Es wird die Elektronenstruktur gewisser binärer ferromagnetischer Legierungen untersucht und mit der Größe ihrer Sättigungsmagnetisierung in Verbindung gebracht. Im innenzentriert kubischen Gitter spalten die 3 d-Atomzustände in zwei energetisch hochliegende schmale Bänder und drei tiefliegende weite Bänder auf. Wenn man annimmt, daß die drei breiten Bänder noch mit $2 \cdot 0,4$ Elektronen pro Atom in die oberen schmalen Bänder hineinragen, dann läßt sich die Größe der beobachteten Sättigungsmagnetisierung aus energetischen Gesichtspunkten heraus verstehen.
\end{abstract}

$\mathrm{D}$ ie nachfolgende Arbeit befaßt sich mit den binären ferromagnetischen Legierungen $\mathrm{Ni}-$ $\mathrm{Cu}, \mathrm{Ni}-\mathrm{Zn}, \mathrm{Ni}-\mathrm{Co}, \mathrm{Fe}-\mathrm{Ni}, \mathrm{Fe}-\mathrm{Co}, \mathrm{Fe}-\mathrm{V}$ und $\mathrm{Fe}-\mathrm{Cr}$. Dabei soll vor allem deren Elektronenstruktur untersucht und mit der Größe der beobachteten Sättigungsmagnetisierung in Verbindung gebracht werden. Die erwähnten zusammenlegierten Elemente nehmen mit Ausnahme von $\mathrm{Cu}$ und $\mathrm{Zn}$ im periodischen System eine gewisse Sonderstellung ein. Bei ihnen sind die Plätze der durch die Hauptquantenzahl 3 bezeichneten Elektronenschale noch nicht alle besetzt, während die vierte Schale bereits angefangen wurde. Schon die freien Atome besitzen im Dampfzustand ein größeres magnetisches Moment. Da die 3-, genauer 3d-Schale, nicht voll besetzt ist, kann ein Teil der Elektronen die Spins parallel richten. Offenbar ist hier der $\mathrm{Zu}$ stand des größtmöglichèn resultierenden Spinmomentes energetisch vor demjenigen mit abgesättigten Spins bevorzugt. Je nachdem wie sich im Kristallverband die Atommomente zusammensetzen, bezeichnet man den Kristall mit einem resultierenden Moment als ferromagnetisch oder beimFehlen eines solchen als antiferromagnetisch. Bei der folgenden Betrachtung sollen die Legierungen nur in denjenigen Temperatur- und Konzentrationsintervallen betrachtet werden, in denen ferromagnetisches Verhalten vorliegt.

Bekanntlich spalten im Kristall die ursprünglich scharfen Atomniveaus in sog. Energiebänder auf. So weiß man, daß in den oben betrachteten Metallen die aus den $3 \mathrm{~d}$ - und $4 \mathrm{~s}$-Atomzuständen hervor- gehenden Bänder sich teilweise überlappen, weshalb ein Teil der $4 \mathrm{~s}$-Elektronen in das $3 \mathrm{~d}$-Band übergeht. So ist es auch zu erklären, daß diese Übergangsmetalle nur 0,6-0,7 $4 \mathrm{~s}$-Elektronen pro Atom besitzen. Überlegungen hinsichtlich der Legierungen scheinen schwieriger, da hierbei verschiedene Atome zum Kristall zusammentreten. Slate $\mathrm{r}^{1}$ wies darauf hin, daß gerade die magnetischen Eigenschaften der erwähnten Legierungen nur von der mittleren Zahl der 3 d-Elektronen abhängen, was wahrscheinlich seinen Grund darin hat, daß sich wenigstens in den weiter unten näher bezeichneten Legierungen die 3 d-Elektronen im Mittel gleichmäßig auf die verschiedenen Atomsorten verteilen. Je nach der prozentualen Zusammensetzung einer Legierung ist jede gewünschte mittlere Besetzung der $3 \mathrm{~d}$-Schale herzustellen. Trägt man die Anzahl der d-Löcher pro Atom, d. h. die in einer vollen d-Schale fehlenden Elektronen, gegen das magnetische Sättigungsmoment pro Atom auf, so erhält man den in Abb. 1 dargestellten Verlauf. Das magnetische Moment nimmt zunächst linear zu, so daß jedes ungepaarte Elektron den vollen Beitrag von einem Bohrschen Magneton liefert. Ab 2,4 Löchern nimmt die Sättigungsmagnetisierung wieder ab. Für jedes weitere aus der $3 \mathrm{~d}$-Schale genommene Elektron fällt das magnetische Moment um ein Bohrsches Magneton. Vom Atom her wäre man eigentlich geneigt anzunehmen, daß das magnetische Moment bei fünf Löchern in der d-Schale seinen Maximal-

\footnotetext{
${ }^{1}$ J. C. Slater, J. appl. Physics, 8, 385 [1937]. ,
} 
wert von fünf Bohrschen Magnetonen erreichen würde, um dann erst bei vollständig ausgeräumter d-Schale denWert Null anzunehmen. Aufgabe dieser Arbeit ist, dieses vorzeitige Umbiegen der Magnetisierungskurve in der Abb. 1 zu erklären.

\section{Die Elektronenstruktur}

Wie Slater ${ }^{1}$ schon bemerkt hat, sind die betrachteten Legierungen bis zu etwa zwei Löchern in der d-Schale kubisch flächenzentriert und bei höheren Löcherkonzentrationen kubisch raumzentriert. Der ,,anomale“ Abfall der Magnetisierungskurve liegt also ganz im Gebiet des innenzentrierten Gitters, so daß wir unsere Untersuchungen auf diesen Gittertyp beschränken wollen. Dabei sollen zunächst ein Elementgitter und weiter unten auch Legierungen betrachtet werden. Die Überlegungen gelten also beispielsweise der innenzentrierten Modifikation des Eisens, die in der Abb. 1 bei einer

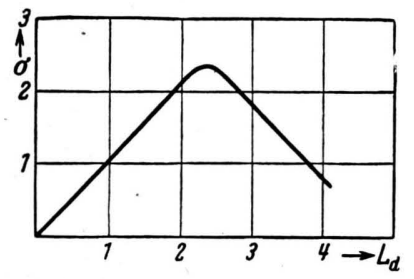

Abb. 1. Verlauf der Sättigungsmagnetisierung $\sigma$, gemessen in Bohrschen Magnetonen pro Atom, in Abhängigkeit von der mittleren Löcherzahl $L_{\mathrm{d}}$ in der $3 \mathrm{~d}-\mathrm{Sch} a \mathrm{le}$ für die Legierungen $\mathrm{Ni}-\mathrm{Cu}, \mathrm{Ni}-\mathrm{Zn}, \mathrm{Ni}-\mathrm{Co}$, $\mathrm{Fe}-\mathrm{Ni}$, Fe-Co, Fe-V und Fe-Cr.

Löcherkonzentration von 2,7 pro Atom mitenthalten ist. Diese Tatsache deutet schon darauf hin, daß in dieser Frage kein wesentlicher Unterschied .zwischen Elementgittern und Legierungen besteht.

Bekanntlich spalten die im Atom entarteten fünf 3 d-Funktionen bei Störungen kubischer Symmetrie in zwei Funktionsfamilien mit verschiedenem Eigenwert auf ${ }^{2,3}$. Diese lauten, wenn man sie in der üblichen Weise nur durch ihren Winkelanteil charakterisiert, $z^{2}-1 / 2\left(x^{2}+y^{2}\right), x^{2}-y^{2}$ und $x y, y z, z x$. Im Kristall haben die Lösungen der SchrödingerGleichung die Form $e^{i \mathfrak{f} \mathfrak{r}} u(\tilde{\mathfrak{r}})$, wobei $\mathfrak{f}$ der sogenannte Ausbreitungsvektor und $u(\tilde{\mathfrak{x}})$ eine Funktion von der Periodizität des Gitters ist. Die erwähnte Zerlegung der fünf $3 \mathrm{~d}$-Funktionen gilt nur streng für solche f́-Vektoren, die sich unter den Symmetrieoperationen der kubischen Gruppe in sich selbst

2 H. A. Bethe, Ann. Physik 3, 133 [1929].

${ }^{3}$ Von der Lage u. H. A. Bethe, Physic. Rev. 71, 612 [1947]. bzw. in solche Vektoren transformieren, die sich nur durch einen Vektor des $2 \pi$-fach vergrößerten reziproken Gitters unterscheiden. Dies sind hier z. B. die Vektoren $^{4} \mathfrak{f}=(0,0,0)$ und $(0,0, \pi / a)$, wenn $2 a$ die Gitterkonstante ist.

Es sollen die 3d-Zustände in der Näherung fast gebundener Elektronen behandelt werden. Wie noch gezeigt wird, kombinieren in dieser Näherung für alle $\mathfrak{f}$-Werte die beiden Funktionsfamilien kaum miteinander. Überdies gilt ein solches Kombinationsverbot auch für die beiden Funktionen $z^{2}-1 / 2\left(x^{2}+y^{2}\right)$ und $x^{2}-y^{2}$ der ersten Funktionsfamilie untereinander. Für die letztgenannten Funktionen wird deshalb auch die Aufspaltung der Niveaus im Gitter leicht zu berechnen sein. Nach bekannten Formeln ${ }^{5}$ liegt der Schwerpunkt der entstehenden Bänder um den Betrag

$$
\varepsilon=\left|\int \psi(\mathfrak{r}) \psi^{*}(\mathfrak{r}) \sum_{l}^{\prime} V\left(\mathfrak{r}-\mathfrak{r}_{l}\right) \mathrm{d} \tau\right|
$$

tiefer als das ungestörte Atomniveau. In Gl. (1) bedeutet $\psi(\mathfrak{r})$ die ursprüngliche Atomfunktion und $\sum_{l}^{\prime} V\left(\mathfrak{r}-\mathfrak{r}_{l}\right)$ das Potential, mit dem die Nachbaratome das herausgegriffene Atom stören. Um die Größe $\varepsilon$ abzuschätzen, betrachten wir die Knotenflächen der beiden Funktionen $z^{2}-1 / 2\left(x^{2}+y^{2}\right)$ und $x^{2}-y^{2}$, die in der Abb. 2 dargestellt sind. Man
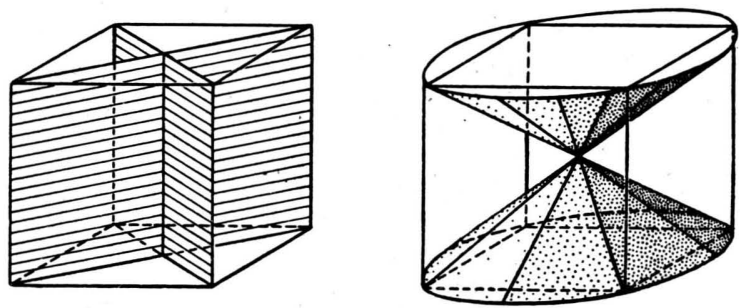

Abb. 2. Lage der Knotenflächen für die beiden Funktionen $x^{2}-y^{2}$ und $z^{2}-1 / 2\left(x^{2}+y^{2}\right)$.

sieht, daß die Verbindungslinien zu den acht nächsten Nachbarn gerade auf Knotenflächen liegen. Der Faktor $\psi(\mathfrak{r}) \psi^{*}(\mathfrak{r})$ in Gl. (1) wird im Gebiet maximalen Störpotentials besonders klein, so daß auch der Wert von $\varepsilon$ relativ klein bleibt.

Anders verhält es sich bei der dreifach entarteten Funktionsfamilie. Im allgemeinen wird die $\psi$-Funktion in der Umgebung eines Atoms durch eine Linearkombination der drei erwähnten Funktionen

4 L. P. Brouckaert, R. Smoluchowski u. E. Wigner, Physic. Rev. 50, 58 [1936].

5 F. Seitz, Modern Theory of Solids S. 303, Mc Graw Hill Book Company, Inc., New York and London 1940 . 
dargestellt werden, die zur Abkürzung mit $F_{1}, F_{2}$ und $F_{3}$ bezeichnet sein mögen,

$$
\psi=A F_{1}+B F_{2}+C F_{3} .
$$

Die Koeffizienten $A, B$ und $C$ sind zunächst unbekannt und können von $\mathfrak{f}$ abhängen. Aus Normierungsgründen ist jedoch

$$
A A^{*}+B B^{*}+C C^{*}=\mathrm{i} .
$$

Bildet man nun (1) und integriert, dann fallen alle gemischten Glieder weg und es bleibt

$$
\begin{aligned}
\varepsilon=\mid A A^{*} & \int F_{1} F_{1}{ }^{*} \sum_{l}^{\prime} V\left(\mathfrak{r}-\mathfrak{r}_{l}\right) \mathrm{d} \tau \\
& +B B^{*} \int F_{2} F_{2}{ }^{*} \sum_{l}^{\prime} V\left(\mathfrak{r}-\mathfrak{r}_{l}\right) \mathrm{d} \tau \\
& +C C^{*} \int F_{3} F_{3} \sum_{l}{ }_{l}^{\prime} V\left(\mathfrak{r}-\mathfrak{r}_{l}\right) \mathrm{d} \tau \mid .
\end{aligned}
$$

Da die drei Integrale einander gleich sind, wird der Wert von $\varepsilon$ wegen Gl. (3) unabhängig von der speziellen Linearkombination und damit auch von $\mathfrak{k}$. Man kann deshalb schreiben

$$
\varepsilon=\left|\int F_{1} F_{1}{ }^{*} \sum_{l}^{\prime} V\left(\mathfrak{r}-\mathfrak{r}_{l}\right) \mathrm{d} \tau\right| .
$$

Bildet man nun (5) mit der Funktion $x y$, so erkennt man, daß derWert hier wesentlich größer ist als für die andere Funktionsfamilie. Die Knotenflächen liegen nicht mehr im Gebiet größten Störpotentials.

$\mathrm{Zu}$ der Energiekorrektion $\varepsilon$ kommt noch eine zweite hinzu, die der Abhängigkeit der Energie vom Ausbreitungsvektor $\mathfrak{i}$ Rechnung trägt. Sie wird durch den Ausdruck

$$
\varrho=\sum_{l}^{\prime} \alpha_{l} e^{i \mathfrak{f r}_{l}}
$$

dargestellt, wobei die Summe über alle nächsten Nachbarn $l$ zu erstrecken ist. In Gl. (6) ist

$$
\alpha_{l}=\int \psi^{*}\left(\mathfrak{r}-\mathfrak{r}_{l}\right) V(\mathfrak{r}) \psi(\mathfrak{r}) \mathrm{d} \tau .
$$

Wenn $\alpha_{l}$ von $l$ und $\mathfrak{f}$ unabhängig ist, dann gilt beim innenzentrierten Gitter

$$
\varrho=8 \alpha \cos k_{x} a \cos k_{y} a \cos k_{z} a .
$$

Hierin sind $k_{x}, k_{y}$ und $k_{z}$ die drei Komponenten des Ausbreitungsvektors und $2 a$ die Gitterkonstante. $\alpha$ ist also ein direktes Maß für die Breite der Bänder. Für die Funktionen der zweifach entarteten Familie sind die zu Gl. (8) erwähnten Forderungen hinsichtlich $\alpha_{l}$ erfüllt. Man erkennt, daß in beiden Fällen $\alpha$ einen kleinen Wert annimmt. Im Bereich maximaler Überlappung zweier benachbarter Atomfunktionen liegen die Knotenflächen (Abb. 2).

Für die andere Funktionsfamilie erscheinen die Überlegungen deshalb schwieriger, weil die $\psi$-Funk- tion durch eine Linearkombination aus den drei Einzelfunktionen dargestellt wird. $\alpha_{l}$ ist nicht mehr von $l$ und $\mathfrak{f}$ unabhängig. Beschränkt man sich darauf, die Breite der Bänder zu errechnen, dann werden die Überlegungen sehr vereinfacht. Wie schon hingewiesen wurde, gilt die Zerlegung in die zwei entarteten Funktionsfamilien streng für die $\mathfrak{f}$-Vektoren $(0,0,0)$ und $(0,0, \pi / \mathrm{a})$. Diese beiden Vektoren gehören aber gerade zum unteren und oberen Bandrand, wie etwa aus Gl. (8) entnommen werden kann. Der Eigenwert an diesen hochsymmetrischen Punkten im $\mathfrak{f}$-Raum kann aber wegen der strengen Entartung nicht mehr von der speziellen Linearkombination der Funktionen $x y, y z$ und $z x$ abhängen, so daß man bei der Berechnung der Energie eine der Funktionen verwenden kann. Für den oberen und unteren Bandrand wird deshalb Gl. (8) verwendbar. Berechnet man $\alpha$ für eine der Funktionen, so ergibt sich ein wesentlich größerer Wert als bei der oben behandelten Funktionsfamilie. Die Funktionen besitzen im Gebiet stärkster Überlappung keine Knotenflächen.

Man kann sich noch überzeugen, daß die Behauptung bezüglich des Nichtkombinierens der Funktionen $z^{2}-1 / 2\left(x^{2}+y^{2}\right)$ und $x^{2}-y^{2}$ mit $x y, y z$ und $z x$ sowie der beiden erstgenannten untereinander zutreffend ist. Zwischen zwei nichtkombinierenden Funktionen $\Phi$ und $\Psi$ muß in dieser Näherung die Beziehung gelten

$$
\int \Phi^{*}(\mathfrak{r}) V\left(\mathfrak{r}-\mathfrak{r}_{l}\right) \Psi\left(\mathfrak{r}-\mathfrak{r}_{l}\right) \mathrm{d} \tau=0 .
$$

Man überzeugt sich leicht, daß Gl. (9) in den erwähnten Fällen durch die besondere Lage der Knotenflächen sehr genähert erfüllt ist.

Im innenzentrierten Gitter spalten also die fünf $3 \mathrm{~d}$-Atomniveaus in je zwei und drei praktisch entartete Bänder auf. Während das zweifach entartete Band energetisch relativ zum ungestörten Atomzustand kaum sinkt und wenig aufspaltet, liegt das dreifach entartete Band tiefer und spaltet auch weit auf (Abb. 3).

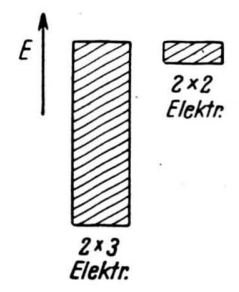

Abb. 3. Energetische Lage der 3d-Bänder im innenzentriert kubischen Gitter. 
In Legierungen sind diese Überlegungen erschwert, weil die verschiedenen Atome ungleiche Besetzungen der 3d-Schale aufweisen und auch die energetische Lage der Atomzustände im allgemeinen verschieden sein wird. Die verschiedene Höhe der Niveaus kann man sich im Kristall in einem ersten Schritt dadurch ausgeglichen denken, daß vom energetisch höheren Zustand Elektronen in das Atom mit dem niedriger liegenden Niveau übertreten. Dies bedeutet eine Angleichung, weil nunmehr im einen Atom die Dichte der Ladungswolke der 3d-Elektronen kleiner und im andern größer geworden ist. Die elektrostatischeWechselwirkungsenergie im Atom wurde im einen Fall verringert und im andern vergrößert. Eine zuverlässige Abschätzung dieser Verhältnisse scheint noch nicht möglich zu sein. Wir sind der Ansicht, daß sich, wenigstens in denjenigen Legierungen, die sich in die Abb. 1 einordnen und im Mittel zwei oder mehr Löcher in der 3d-Schale haben, die ursprünglich unterschiedlichen Besetzungszahlen der verschiedenen Atomsorten genähert angleichen. Eine Stütze dieser Überlegung ist die Tatsache, daß die Sättigungsmagnetisierung nur von der mittleren Zahl der dElektronen und nicht von der speziellen Atomzusammensetzung der Legierung abhängt. Nach diesem ersten Schritt ist eine Störungsrechnung ähnlich der beim Elementgitter durchzuführen. Obwohl dieVerteilung der Quantenzustände qualitativ durch die Abb. 3 wiedergegeben wird, so steht trotzdem eine genauere Berechnung der Eigenfunktionen und Quantenenergien in einem solchen Mischkristall noch aus. Für die folgenden Betrachtungen ist jedoch die Anwendbarkeit des Bandmodelles und damit das in Abb. 3 dargestellte Ergebnis von entscheidender Bedeutung.

\section{Energetische Betrachtungen}

In diesem Abschnitt wollen wir die Energie des Kristalles in Funktion seines magnetischen Gesamtmomentes betrachten. Einen ersten Teil dieser Energie kann man dem Bänderschema entnehmen. Im nichtferromagnetischen Fall ist bekanntlich jeder Quantenzustand durch zwei Elektronen mit antiparallelen Spins besetzt. Um ein resultierendes Spinmoment zu erzeugen, müssen Elektronen aus - den doppelt besetzten Zuständen herausgenommen und mit umgedrehten Spins in den nächsthöheren noch freien Quantenplätzen untergebracht werden. Hierbei ist Arbeit zu leisten. Um deren Größe ge- nauer berechnen zu können, ist eine Kenntnis der energetischen Dichte der Quantenzustände erforderlich. Für die nachfolgenden Untersuchungen ist es aber schon ausreichend, zu wissen, daß deren Dichte in dem breiten dreifachen Band wesentlich kleiner ist als in dem schmalen Doppelband. Wir verzichten bewußt auf eine genaue Struktur, indem wir die Elektronendichte schematisch in der Abb. 4 dar-

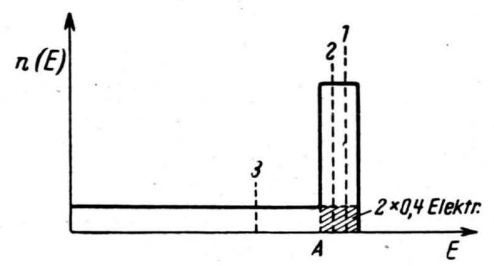

Abb. 4. Idealisierter Verlauf der Zustandsdichte $n(E)$ in den 3d-Bändern des innenzentriert kubischen Gitters in Abhängigkeit von der Energie $E$.

stellen. In diesem Diagramm ist einfachheitshalber angenommen, daß der obere Rand beider Bänder zusammenfällt. Die Überlegungen sind jedoch von dieser Annahme unabhängig. Es sollen nur beide Bänder überlappen. Wie weiter unten noch gezeigt wird, gelangt man zu Übereinstimmung mit den Experimenten, wenn man annimmt, daß das untere Band noch mit 2 0,4 Elektronen pro Atom in das obere schmale Band hineinragt. Im folgenden sollen drei Fälle diskutiert werden.

Die 3d-Bänder seien bei vollständig abgesättigten Spins bis zu der in Abb. 4 mit 1 bezeichneten Stelle besetzt. Bei der Erzeugung eines magnetischen Momentes muß man, wie schon hingewiesen, Elektronen aus den obersten doppelt besetzten Quantenzuständen herausnehmen und sie mit umgedrehtem Spin in die nächsthöheren noch freien Zustände heben. Die dabei aufzuwendende Energie ist relativ klein, weil die Energiezustände sehr dicht liegen. Schließlich erreicht man einen Zustand, in dem das Band der Abb. 4 kein vollständig freies Niveau mehr hat. Eine weitere Vergrößerung des magnetischen Momentes ist dann nicht mehr möglich. Die Energiezunahme bei wachsendem Spinmoment ist in der Abb. 5a durch die ausgezogene Kurve dargestellt.

Geht man von der durch 2 markierten Besetzung aus, dann muß man schließlich bei einem größeren magnetischen Moment auch Elektronen aus dem Gebiet herausnehmen, das in der Abb. 4 links von der mit A bezeichneten Stelle liegt. Da hier die Quantenzustände viel weniger dicht beieinanderliegen, nimmt die aufzuwendende Energie sehr viel schneller zu als in dem Gebiet der hohen Zustands- 

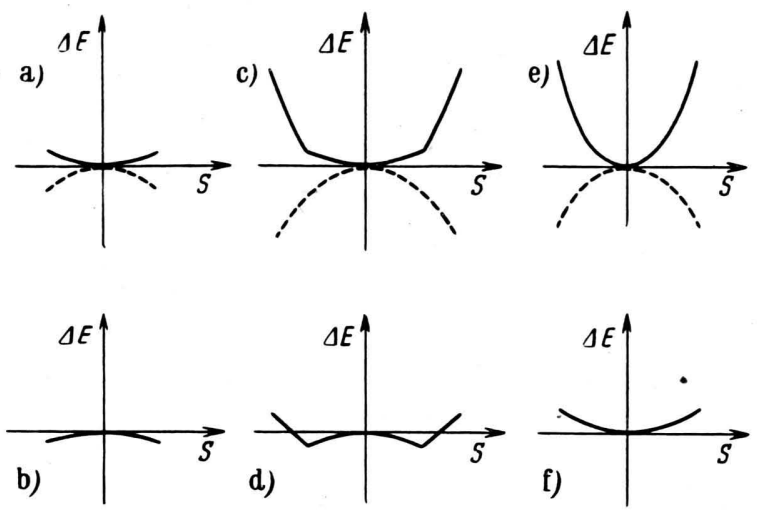

Abb. 5. In den Abb. 5a, c und d ist die Energiezunahme $\Delta E$ nach dem Bandmodell (ausgezogen) und die Änderung der Austauschenergie (gestrichelt) bei drei verschiedenen Elektronenkonzentrationen in der $3 \mathrm{~d}$-Schale gegen das resultierende Spinmoment qualitativ aufgetragen.

Die Abb. 5 b, $d$ und $f$ stellen die Summe beider Energiebeiträge dar.

dichte. Man kann sich davon überzeugen, daß die Energiekurve einen Knick hat, wenn gerade alle Elektronen in dem dichtbesetzten „Band“ gleichen Spin haben (Abb. 5c).

Bei der durch 3 markierten Besetzung befindet man sich ganz im Gebiet der weiten Bänder. Wegen der geringen Dichte der Elektronenzustände ist die Energiezunahme bei wachsendem Spinmoment stets groß (Abb. 5e).

Alle weiteren spinabhängigen Energieanteile pflegt man unter dem Namen Austauschenêrgie zusammenzufassen. Wir wollen uns nicht auf detaillierte Vorstellungen hinsichtlich dieses Energiebeitrages festlegen, sondern hierzu lediglich einige Feststellungen treffen: Im ferromagnetischen Kristall ist die Austauschenergie bei einem resultierenden Spinmoment negativ. Denkt man sich dieses Spinmoment variabel, dann nimmt im allgemeinen der Betrag der Austauschenergie bei wachsendem resultierendem Spinmoment zu. Dies kann zwar in solchen Legierungen anders sein, in denen sich die einzelnen Atomsorten sehr verschieden verhalten, also z. B. eine ausgesprochen starke Tendenz zeigen, ihre Atomspins antiparallel einzustellen. Unter diesen Umständen wäre aber auch eine einfache Anwendung des Bandmodells nicht mehr am Platze. Der qualitative Verlauf der Austauschenergie ist in den Abb. 5a, c und e gestrichelt eingezeichnet. Es ist schwer, die relative Größe des positiven und negativen Energiebeitrages $\mathrm{zu}$ bestimmen. Abschätzungen ergeben lediglich, daß beide von der gleichen Größenordnung sind. Die positive Energiekurve besitzt im allgemeinen einen flachen und steilen Teil, wie oben schon erläutert wurde. Wir wollen annehmen, daß wenigstens in dem flachen Gebiet die negative Austauschenergie größer ist als der positive Energiebeitrag. Dies bedeutet, daß im flachen Teil der Zustand höchsten Spinmomentes begünstigt ist (Abb. 5b). Sind also weniger als 2,4 Löcher in der d-Schale, dann richten sich die Spins aller ungepaarten Elektronen gleich. Bei größeren Löcherzahlen aber hat die positive Energiekurve einen Knick. An dieser Stelle stoßen der flache und steile Teil der Kurve zusammen. Wenn der Knick hinreichend ausgeprägt ist, dann besitzt die Kurve der Gesamtenergie an dieser Stelle ein Minimum (Abb. 5d). Es ist jetzt nicht mehr der Zustand des höchstmöglichen Spinmomentes energetisch begünstigt. Offenbar sind nur diejenigen Quantenzustände mit ungepaarten Elektronen parallelen Spins besetzt, die im Gebiet des schmalen „Bandes“, d. h. in Abb. 4 rechts von der Stelle A liegen. Beim Herausnehmen weiterer Elektronen wird das schmale „Band“ immer mehr ausgeräumt und das magnetische Moment nimmt ab. Schließlich kommt man $\mathrm{zu}$ dem in Abb. 5e und $f$ dargestellten Fall. Hier ist das d-Band bei abgesättigten Spins bis zu der in Abb. 4 mit 3 bezeichneten Stelle besetzt. Wie man sieht, wird die beim Gleichrichten der Spins aufzuwendende Energie nicht mehr von der negativen Austauschenergie kompensiert. Der energetisch günstigste Zustand liegt beim magnetischen Moment Null.

Mit Hilfe der entwickelten Vorstellungen kann man also die Abb. 1 vollständig deuten. Bei Löcherzahlen unter 2,4 pro Atom stellt sich das höchstmögliche Spinmoment ein. Jedes Loch liefert einen Beitrag von einem Bohrschen Magneton. Bei größeren Löcherzahlen dagegen wird das schmale „Band“, das dann nur noch von Elektronen parallelen Spins besetzt ist, ausgeräumt, so daß für jedes weitere Loch das magnetische Moment wieder um ein Bohrsches Magneton abnimmt.

In unseren Betrachtungen mußten wir nicht von der hergebrachten Bandvorstellung abweichen. In älteren Überlegungen ${ }^{6}$ bildete die Annahme, daß der Quotient aus der Gesamtzahl der Quantenzustände pro Band und der der Atome in den betrachteten Gittern nicht ganzzahlig sein soll, eine gewisse Schwierigkeit.

${ }^{6}$ L. Pauling, Physic, Rev. 54; 899 [1938]. 
Eine erst jetzt erschienene Arbeit von Zener? greift das oben behandelte Problem von einer anderen Seite an. Die entwickelte Theorie bedarf noch einer experimentellen Prüfung.

Eine Arbeit von $\mathrm{Carr}^{8}$ behandelt die ferromagnetischen Legierungen vom Standpunkt der

7 C. Zener, Physic. Rev. 85, 324 [1952].

${ }^{8}$ W. J. Carr jr., Physic. Rev. 85, 590 [1952].
Heitler-London-Näherung. Bei den hier nicht betrachteten Legierungen $\mathrm{Ni}-\mathrm{V}, \mathrm{Ni}-\mathrm{Cr}, \mathrm{Ni}-\mathrm{Mn}, \mathrm{Co}-$ Mn und Co-Cr scheint diese Näherung angemessen zu sein. Bei den übrigen Legierungen scheinen beide Auffassungen die experimentellen Ergebnisse wiedergeben zu können.

Für Diskussionen und Ratschläge möchte der Verfasser Herrn Prof. Dr. E. Fues seinen Dank aussprechen.

\title{
Struktur des amorphen Selens
}

\author{
Von H. Richter, W. Kulcke und H. Specht \\ Aus dem Röntgeninstitut der Technischen Hochschule, Stuttgart, und dem Institut für \\ Metallphysik am Max-Planck-Institut für Metallforschung, Stuttgart \\ (Z. Naturforschg. 7 a, 511-532 [1952]; eingegangen am 7. April 1952)
}

\begin{abstract}
Die hier untersuchten amorphen Se-Präparate wurden durch Aufdampfen bzw. chemische Fällung oder durch plötzliches Abkühlen einer Se-Schmelze hergestellt. Die Atomabstände und Atomzahlen in den verschiedenen Se-Proben wurden durch Fourier-Analyse bestimmt. Ähnlich wie beim amorphen As wird auch hier in starker Anlehnung an das zugehörige Gitter ein Strukturmodell für das amorphe Se entwickelt. In diesem Modell besitzen die Se-Ketten praktisch denselben Bau und die gleiche Lagerung wie im Gitter, nur sind ihre gegenseitigen Abstände erheblich größer. Abweichend vom Gitter nehmen die Se-Atome in diesem Kettenmodell keine festen Raumlagen ein, sondern sind um diese, vornehmlich in Richtung der Kettenachse statistisch regellos verteilt. Die aus diesem Strukturmodell errechneten Atomabstände stimmen mit den beobachteten bestens überein. In allen untersuchten Se-Präparaten liegt dieselbe Kettenstruktur vor; verschiedene Formen des amorphen Zustandes wurden hier nicht beobachtet. Die Kettenstruktur von Se entspricht der Stäbchenstruktur von sublimiertem (amorphem) As.

Der Übergang amorph $\rightarrow$ kristallin wurde durch Tempern der Se-Proben erreicht. Die Aufnahmen zeigen, daß die Umwandlung gebietsweise stattfindet, so daß noch völlig amorphe Bezirke neben bereits kristallisierten vorliegen, vielleicht im Rahmen ein und desselben Atomverbandes. Die Umwandlung des amorphen Se in die zugehörige kristalline Struktur erfolgt in den einzelnen Bereichen plötzlich und bei verschiedenen Temperaturen, ohne daß dabei irgendwelche Zwischenstrukturen durchlaufen werden.
\end{abstract}

$\mathrm{D}^{2}$ as glasige (amorphe) Se wurde mehrfach röntgenographisch untersucht. So fanden Briegleb $^{1}$ und Tanaka ${ }^{2}$ an glasigem Se verbreiterte Interferenzen. Tanaka beobachtete beim Erwärmen von glasigem Se neben den amorphen auch einzelne kristalline Ringe des metallischen (hexagonalen) Se. Nach fünfstündigem Tempern bei $200^{\circ} \mathrm{C}$ waren nur noch die kristallinen Ringe von hexagonalem Se festzustellen; Ähnliches fanden Prins und Dekey ser ${ }^{3}$. Durch Fourier-Analyse der Streukurve konnten

${ }^{1}$ G. Briegleb, Z. physik. Chem. Abt. A 144, 321 [1929].

2 K. Tanaka, Mem. Coll. Sci., Kyoto, Imp. Univ. 17, 59 [1934].

${ }^{3}$ J. A. Prins u. W. Dekeyser, Physica, 4, 900 [1937].

${ }^{4}$ K. Lark-Horovitz u. E. P. Miller, Amer. phy-
Lark-Horovitz und Miller ${ }^{4}$ erstmals Aussagen über die bevorzugten Atomabstände im glasigen Se machen. Nach der gleichen Methode bestimmte Hendus ${ }^{5}$ die Atomabstände sowie die Koordinationszahlen im glasartigen Se. Er bestätigte die von Lark-Horovitz und Miller gefundenen Atomabstände. In der $\mathrm{Z}$ wischenzeit ist durch die Arbeiten von Richter und Fürst ${ }^{6}$ sowie Richter und Breitling ${ }^{7}$ die Aufnahme- und Auswertungstechnik wesentlich verbessert worden.

sic. Soc. 11, 13 [1936]; Physic. Rev. 51, 380 [1937]; Nature [London] 146, 459 [1940].

${ }^{5}$ H. Hendus, Z. Physik 119, 278 [1942].

${ }^{6}$ H. Richter u. O. Fürst, Z. Naturforschg. 6a, 38 [1951].

${ }^{7}$ H. Richter u. G. Breitling, Z. Naturforschg. 6a, 721 [1951]. 